\title{
Knockdown of Nedd8-conjugating enzyme UBE2M suppresses the proliferation and induces the apoptosis of intrahepatic cholangiocarcinoma cells
}

\author{
BIN ZHAO*, CAIYAN GAO*, DAIXIU SHI*, JIE MAO*, JUN ZHAO, \\ LINGYUN GUO, JIWU GUO and ZUOYI JIAO
}

Department of General Surgery, Lanzhou University Second Hospital, Lanzhou, Gansu 730030, P.R. China

Received January 7, 2019; Accepted June 21, 2019

DOI: $10.3892 /$ or.2019.7327

\begin{abstract}
As an important regulator of neddylation, neural precursor cell expressed developmentally downregulated 8 (Nedd8)-conjugating enzyme E2M (UBE2M) mediates cullin neddylation. Upregulation of the neddylation pathway is associated with tumor progression in intrahepatic cholangiocarcinoma (ICC). The present study was designed to assess the effects of Nedd8-conjugating enzyme UBE2M knockdown on intrahepatic cholangiocarcinoma cells, and to determine the potential underlying mechanisms. UBE2M and associated protein expression levels were determined via immunohistochemistry and western blotting. ICC cells were transfected with short hairpin RNA to knockdown UBE2M expression. Cell Counting Kit-8 and colony formation assays, and xenograft experiments were used to examine cell viability and colony survival in vitro, and tumor formation in vivo. Survival was evaluated using Kaplan-Meier analysis and log-rank tests. Patients with ICC presenting high expression of UBE2M exhibited worse accumulative recurrence and overall survival compared with patients with low expression. Knockdown of UBE2M expression led to a decrease in the viability and clonogenic survival of QBC939 and HUCCT1 cells, and suppressed tumor formation in vivo. UBE2M silencing caused accumulation of cullin-RING ligase substrates (chromatin-licensing and DNA replication factor 1 and origin recognition complex subunit 1), inducing DNA damage responses and apoptosis. The present findings suggested that UBE2M serves an important role in ICC progression and may present as a novel target for the treatment of ICC.
\end{abstract}

Correspondence to: Dr Zuoyi Jiao, Department of General Surgery, Lanzhou University Second Hospital, 82 Cuiying State, Chengguan, Lanzhou, Gansu 730030, P.R. China

E-mail: jiaozuoyi@163.com

*Contributed equally

Key words: intrahepatic cholangiocarcinoma, neural precursor cell expressed developmentally downregulated 8, NEDD8-conjugating enzyme E2M, ubiquitination, tumor apoptosis

\section{Introduction}

Dysregulation of the ubiquitin-proteasome pathway serves an important role in tumor development and progression (1). As the largest family of multiunit E3 ubiquitin ligases (crucial control points in the ubiquitin-proteasome pathway), cullin-RING ligases (CRLs) target numerous ubiquitinated protein substrates for proteasome-targeted degradation $(2,3)$. Importantly, CRL activation requires cullin neddylation $(4,5)$, an ubiquitination modification process in which the ubiquitin-like protein neural precursor cell expressed developmentally downregulated 8 (NEDD8) is conjugated to cullins (4-8). The process involves NEDD8-activating enzyme E1 (NAE1), NEDD8-conjugating enzyme E2M (UBE2M) and E3 NEDD8 ligases $(6,9)$. UBE2M interacts with a specific E3 ubiquitin ligase to promote the neddylation of cullins (10), thereby regulating cell cycle checkpoint control, DNA damage response, apoptosis and senescence via diverse pathways. As an essential component of neddylation, UBE2M is the critical factor in the progression of various human carcinomas, including human renal cell carcinoma and lung cancers, and intrahepatic cholangiocarcinoma (ICC) (11-14).

According to the Surveillance, Epidemiology and End Results program, ICC is one of the most common liver cancers in the US, accounting for $\sim 15 \%$ of primary liver cancers (15). The incidence and mortality of ICC are gradually increasing worldwide (16). Additionally, due to its frequently asymptomatic nature, patients with ICC are usually diagnosed with late-stage disease, preventing curative treatment and resulting in worse prognosis $(17,18)$. Therefore, effective therapies are urgently required to improve outcomes. MLN4924 is an NAE inhibitor that blocks NAE activity and efficiently suppresses cullin neddylation, inhibiting the growth of ICC cells in vitro and in vivo (14). However, UBE2M, another critical neddylation regulator is poorly understood. In the present study, the roles and underlying mechanisms of UBE2M in ICC were investigated for the first time, to the best of our knowledge.

\section{Materials and methods}

Patients and tissue specimens. ICC tissue samples were obtained from 81 patients with a pathological diagnosis of 
primary ICC that underwent radical resection for their tumor in Lanzhou University Second Hospital between March 2009 and August 2017. Adjacent matched non-cancerous livers were obtained from an incision $>5 \mathrm{~cm}$ away from the carcinoma sites. A total of 29 female and 52 male patients ranging 46-75 years old, who had not received distant metastases nor any preoperative anticancer therapy. Furthermore, for reverse transcription-quantitative PCR (RT-qPCR) analysis, normal intrahepatic bile duct were obtained from 12 patients (5 female and 7 male individuals ranging 43-74 years old), and ICC tissues were obtained from 46 patients (26 male and 20 female patients ranging 33-75 years old) at Lanzhou University Second Hospital between June 2016 and July 2018. A protocol for use of human surgical samples was approved by the Lanzhou University Second Hospital Ethics Committee, and informed consent was obtained from each patient.

The clinicopathological characteristics of patients with ICC are presented in Table I. Outpatient follow-up procedures were performed via letter and telephone, and are presented in Table II. The median follow-up period of the 81 patients was 18.5 months (range, 3.5-90.5 months; SD, 19.3 months). The interval between the date of surgery and the date of first relapse was defined as the time to relapse (TTR); for patients without relapse, the interval between the date of surgery and the end of the follow-up period was recorded. Patients without relapse were censored at the date of the last follow-up. Systemic chemotherapy was given to patients with recurrence; the main recurrence sites were the hilar and bile duct.

Immunohistochemistry (IHC). ICC tissue samples obtained from representative regions and noncancerous liver tissue were stained via IHC. An arraying machine (Beecher Instruments, Inc.) was used to construct tissue microarrays. The tissues were fixed in $10 \%$ formalin neutral buffer solution for $24 \mathrm{~h}$ at room temperature. Following deparaffinization in xylene and rehydration in a graded ethanol series, ICC tissue array sections (4-5 $\mu$ m thick) were treated with $1 \% \mathrm{H}_{2} \mathrm{O}_{2}$ to block endogenous peroxidase activity and antigen retrieval was performed with $0.01 \%$ citrate buffer ( $\mathrm{pH}$ 6.0) for $10 \mathrm{~min}$ in a microwave oven. Rabbit anti-UBE2M monoclonal primary antibodies (1:100; cat. no. ab109507; Abcam) were added and incubated for $30 \mathrm{~min}$ at room temperature. Then, the tissues washed with TBS and incubated with the secondary antibodies $(1: 100$; cat. no. SPN-9001; OriGene Technologies, Inc.) for $2 \mathrm{~h}$ at room temperature. Following visualization of protein expression with 3,3'-diaminobenzidine (DAB), sections were counterstained with hematoxylin at room temperature for $5 \mathrm{~min}$, and comparisons were performed between tumor/normal pairs. Photos were acquired under a Nikon Optiphot microscope equipped with an Optronics charge-coupled-device camera (magnification, x200; Nikon Corporation); five random fields per sample were analyzed. Immunostaining intensity was processed with Image-Pro Plus version 4.1 software (Media Cybernetics, Inc.) and scored as follows: No staining (0), light brown (1), brown (2), and dark brown (3), and scores of 2 and 3 were classified as high expression, whereas scores of 0 and 1 were classified as low expression in prognostic analyses.

RT-qPCR analysis. TRIzol ${ }^{\circledR}$ reagent (Invitrogen; Thermo Fisher Scientific, Inc.) supplemented with RNase-free DNase was used to extract total RNA from 46 ICC tissues and 12 normal intrahepatic bile duct tissues, as well as HUCCT1 and QBC939 cells. A PrimeScript ${ }^{\mathrm{TM}}$ RT reagent kit (Takara Bio, Inc.) was used to perform RT according to the manufacturer's protocols. Then, qPCR was performed on an ABI 7500 thermocycler (Applied Biosystems; Thermo Fisher Scientific, Inc.) using Power SYBR Green PCR MasterMix (Applied Biosystems; Thermo Fisher Scientific, Inc.) as follows: Initial step at $94^{\circ} \mathrm{C}$ for $5 \mathrm{~min}$, followed by 40 cycles of denaturation at $94^{\circ} \mathrm{C}$ for $30 \mathrm{sec}$, annealing at $55-60^{\circ} \mathrm{C}$ for $15 \mathrm{sec}$ and extension at $72^{\circ} \mathrm{C}$ for $60 \mathrm{sec}$, with a final extension at $72^{\circ} \mathrm{C}$ for $7 \mathrm{~min}$. $\beta$-actin was used as endogenous control, and the $2^{-\Delta \Delta \mathrm{Cq}}$ method was performed to measure the expression of target genes (19). The sequences of the primers used are as follows: Human $\beta$-actin, forward 5'-TGACGTGGACATCCG CAAAG-3', reverse 5'-CTGGAAGGTGGACAGCGAGG-3'; human UBE2M, forward 5'-ATGAGGGCTTCTACAAGA GTGG-3', reverse 5'-ATTGTCTCACACTTCACCTTGG-3'.

Cell lines and culture. Human cholangiocarcinoma cell lines QBC939 and HUCCT1 were obtained from the Third Military Medical University (Chongqing, China) and the Cell Resource Center of Tohoku University, respectively. 293T cells were obtained from the American Type Culture Collection. Cells were cultured in Dulbecco's modified Eagle's medium (HyClone; GE Healthcare Life Sciences) supplemented with $10 \%$ fetal bovine serum (FBS; Biochrom AG; Merck KGaA), $1 \%$ penicillin and streptomycin (Corning Inc.) at $37^{\circ} \mathrm{C}$ and $95 \%$ humidity with $5 \% \mathrm{CO}_{2}$.

UBE2M short hairpin (sh)RNA and transfection. The target sequence for the UBE2M shRNA was 5'-GGGCUUCUA CAAGAGUGGGAAGUUU-3' (Invitrogen; Thermo Fisher Scientific, Inc). The sequence of shUBE2M was designed according to a previous study (11). pGC-fu-EGFP (Shanghai GeneChem Co., Ltd.) double-digested by the restriction enzymes EcoRI (cat. no. R0101S; New England BioLabs, Inc.) and BamHI (cat. no. R0136S; New England BioLabs, Inc.) was used as a vector. Positive lentiviral pGC-fu-EGFP vectors with shUBE2M were packaged with pHelper1.0 and pHelper 2.0 vectors (Shanghai GeneChem Co., Ltd.) into 293T cells $\left(4 \times 10^{7}\right.$ cells/dish) using Lipofectamine ${ }^{\circledR} 2000$ (Invitrogen; Thermo Fisher Scientific, Inc.), and the virus were collected and centrifuged at $4,000 \mathrm{x}$ g for $10 \mathrm{~min}$ at $4^{\circ} \mathrm{C}$ after transfection for $48 \mathrm{~h}$. Then the virus was filtrated with a $0.45-\mu \mathrm{m}$ filter and $500 \mu \mathrm{l}$ virus was infected into QBC939 or HUCCT1 cells $\left(5 \times 10^{5}\right.$ cells/well) in DMEM supplemented with $10 \%$ FBS and polybrene at a titer of $3 \times 10^{8} \mathrm{TU} / \mathrm{ml}$, using Lipofectamine 2000, whereas empty vector pGC-fu-EGFP was used as an empty control (shNC). Following infection for $96 \mathrm{~h}$, puromycin was added to the medium at $4 \mu \mathrm{g} / \mathrm{ml}$ and cultured for $48 \mathrm{~h}$ after transfection, and then images were captured.

Cell viability and colony formation assays. Cells transfected with shUBE2M or shNC at a density of 1,500 cells/well were plated into 96-well plates in triplicate. A Cell Counting Kit-8 assay (CCK-8; Beyotime Institute of Biotechnology) was used to determine the cell viability at $48 \mathrm{~h}$ following the aforementioned 96-h transfection period according to the manufacturer's protocols. Following incubation with $10 \mu \mathrm{l}$ CCK-8 reagent for 
Table I. Associations between UBE2M expression and clinicopathologic characteristics in patients with intrahepatic cholangiocarcinoma.

\begin{tabular}{|c|c|c|c|}
\hline \multirow{2}{*}{$\begin{array}{l}\text { Clinicopathological } \\
\text { factors }\end{array}$} & \multicolumn{2}{|c|}{$\begin{array}{c}\text { UBE2M } \\
\text { expression }\end{array}$} & \multirow[b]{2}{*}{ P-valu } \\
\hline & Low & High & \\
\hline Age (years) & & & 0.586 \\
\hline$<61$ & 16 & 25 & \\
\hline$\geq 61$ & 18 & 22 & \\
\hline Sex & & & 0.698 \\
\hline Female & 13 & 16 & \\
\hline Male & 21 & 31 & \\
\hline HBsAg & & & 0.184 \\
\hline Negative & 19 & 33 & \\
\hline Positive & 15 & 14 & \\
\hline Serum AFP (ng/ml) & & & $0.130^{\mathrm{a}}$ \\
\hline$<20$ & 33 & 40 & \\
\hline$\geq 20$ & 1 & 7 & \\
\hline Serum CA 19-9 (ng/ml) & & & 0.888 \\
\hline$<36$ & 15 & 20 & \\
\hline$\geq 36$ & 19 & 27 & \\
\hline Serum ALT (U/l) & & & 0.306 \\
\hline$<40$ & 26 & 31 & \\
\hline$\geq 40$ & 8 & 16 & \\
\hline Serum ALP (U/1) & & & $0.072^{\mathrm{a}}$ \\
\hline$<145$ & 29 & 31 & \\
\hline$\geq 145$ & 5 & 16 & \\
\hline Serum CEA $(\mu \mathrm{g} / \mathrm{l})$ & & & 0.901 \\
\hline$<5$ & 20 & 27 & \\
\hline$\geq 5$ & 14 & 20 & \\
\hline gGGT (U/1) & & & 0.191 \\
\hline$<60$ & 18 & 18 & \\
\hline$\geq 60$ & 16 & 29 & \\
\hline Child-Pugh score & & & $0.130^{\mathrm{a}}$ \\
\hline A & 33 & 40 & \\
\hline B & 1 & 7 & \\
\hline Liver cirrhosis & & & $0.342^{\mathrm{a}}$ \\
\hline No & 27 & 42 & \\
\hline Yes & 7 & 5 & \\
\hline Tumor size $(\mathrm{cm})$ & & & 0.599 \\
\hline$<5$ & 15 & 18 & \\
\hline$\geq 5$ & 19 & 29 & \\
\hline Tumor number & & & 0.530 \\
\hline Single & 28 & 36 & \\
\hline Multiple & 6 & 11 & \\
\hline Microvascular/bile duct invasion & & & 0.532 \\
\hline No & 26 & 33 & \\
\hline Yes & 8 & 14 & \\
\hline Lymphatic metastasis & & & 0.300 \\
\hline No & 22 & 25 & \\
\hline Yes & 12 & 22 & \\
\hline
\end{tabular}

Table I. Continued.

\begin{tabular}{lrrr}
\hline & \multicolumn{2}{c}{$\begin{array}{c}\text { UBE2M } \\
\text { expression }\end{array}$} & \\
\cline { 2 - 3 } $\begin{array}{l}\text { Clinicopathological } \\
\text { factors }\end{array}$ & Low & High & P-value \\
\hline $\begin{array}{l}\text { Tumor encapsulation } \\
\text { Complete }\end{array}$ & 2 & 9 & $0.108^{\mathrm{a}}$ \\
$\quad$ None & 32 & 38 & \\
Tumor differentiation & & & 0.124 \\
$\begin{array}{l}\text { Poor } \\
\text { Moderate/well }\end{array}$ & 19 & 34 & \\
\hline
\end{tabular}

${ }^{\mathrm{a}}$ Fisher's exact test; all other tests are $\chi^{2}$ tests. AFP, $\alpha$-fetoprotein; ALT, alanine aminotransferase; CA 19-9, carbohydrate antigen 19-9; CEA, carcinoembryonic antigen; $\gamma \mathrm{GGT}, \gamma$-glutamyltranspeptidase; $\mathrm{HBsAg}$, hepatitis B surface antigen; UBE2M, UBE2M, neural precursor cell expressed developmentally downregulated 8-conjugating enzyme E2M.

$4 \mathrm{~h}$, the absorbance was measured at $450 \mathrm{~nm}$ using an automatic multi-well spectrophotometer (Bio-Rad Laboratories, Inc.).

For clonogenic assays, 200 cells/well were seeded in 60-mm petri dishes in triplicate. Following incubation for 12 days at $37^{\circ} \mathrm{C}$, colonies were fixed with $4 \%$ paraformaldehyde for $15 \mathrm{~min}$ and stained with $0.5 \%$ crystal violet for $15 \mathrm{~min}$ (both at room temperature). The number of colonies with $>50$ cells was counted using a fluorescence microscope (magnification, x40; Olympus Corporation).

Apoptosis analysis. A fluorescence microscope (magnification, x400; Olympus Corporation) was used to analyze apoptosis in at least five fields per sample at $48 \mathrm{~h}$ after cell seeding in 6-well plates at $2.0 \times 10^{6}$ cells/well.

Propidium iodide (PI) staining and analysis. Harvested cells were fixed with $70 \%$ ethanol at $-20^{\circ} \mathrm{C}$ overnight. Following two washes with ice-cold PBS, cells were stained with PI $(36 \mu \mathrm{g} / \mathrm{ml}$; Sigma-Aldrich; Merck KGaA) containing RNase $(10 \mu \mathrm{g} / \mathrm{ml}$; Sigma-Aldrich; Merck KGaA) in the dark at $37^{\circ} \mathrm{C}$ for $15 \mathrm{~min}$, then a CyAn ${ }^{\mathrm{TM}}$ ADP flow cytometer (Beckman Coulter, Inc.) was used to analyze apoptosis. Apoptosis was determined by the population percentage of cells in sub-G1. Data analysis was performed using ModFit LT 5.0 software (Verity Software House).

Protein extraction and western blotting. Cellular proteins were extracted using cell lysis buffer (Beyotime Institute of Biotechnology), then the concentration was measured with a Pierce $^{\mathrm{TM}}$ BCA protein assay kit (Thermo Fisher Scientific, Inc.). Protein $(50 \mu \mathrm{g})$ was separated via $12 \%$ SDS-PAGE and then transferred to PVDF membranes (EMD Millipore). Membranes were blocked with $5 \%$ skim milk at room temperature for $60 \mathrm{~min}$, and then incubated at $4^{\circ} \mathrm{C}$ overnight with the following primary antibodies: NEDD8 (1:1,000; cat. no. ab81264; Abcam); UBE2M (1:1,000; cat. no. ab109507; Abcam); origin recognition complex subunit 1 (ORC1; 1:1,000; 
Table II. Univariate and multivariate analyses of prognostic factors in patients with intrahepatic cholangiocarcinoma.

A, Univariate analysis

\begin{tabular}{|c|c|c|c|c|}
\hline \multirow[b]{2}{*}{ Variable } & \multicolumn{2}{|l|}{ RFS } & \multicolumn{2}{|l|}{ OS } \\
\hline & $\mathrm{HR}(95 \% \mathrm{CI})$ & P-value & $\mathrm{HR}(95 \% \mathrm{CI})$ & P-value \\
\hline Age $(\geq 61$ years vs. $<61$ years $)$ & $1.208(0.735-1.985)$ & 0.457 & $1.131(0.560-1.966)$ & 0.664 \\
\hline Sex (male vs. female) & $0.737(0.442-1.229)$ & 0.243 & $0.623(0.355-1.094)$ & 0.099 \\
\hline HBsAg (positive vs. negative) & $0.346(0.192-0.622)$ & $<0.001^{\mathrm{a}}$ & $0.258(0.130-0.512)$ & $<0.001^{\mathrm{a}}$ \\
\hline Serum AFP ( $\geq 20 \mathrm{ng} / \mathrm{ml}$ vs. $<20 \mathrm{ng} / \mathrm{ml})$ & $1.182(0.822-3.995)$ & 0.141 & $1.859(0.834-4.143)$ & 0.129 \\
\hline Serum CA 19-9 ( $\geq 36 \mathrm{ng} / \mathrm{ml}$ vs. $<36 \mathrm{ng} / \mathrm{ml})$ & $1.589(0.954-2.646)$ & 0.075 & $2.308(1.261-4.226)$ & $0.007^{\mathrm{a}}$ \\
\hline $\operatorname{Serum~ALT~}(\geq 40 \mathrm{U} / 1 \mathrm{vs} .<40 \mathrm{U} / 1)$ & $1.073(0.626-1.840)$ & 0.798 & $1.184(0.655-2.141)$ & 0.557 \\
\hline Serum ALP ( $\geq 145$ U/1 vs. $<145$ U/l) & $1.430(0.826-2.475)$ & 0.202 & $1.806(1.006-3.244)$ & $0.048^{\mathrm{a}}$ \\
\hline $\operatorname{Serum~CEA~}(\geq 5 \mu \mathrm{g} / 1 \mathrm{vs} .<5 \mu \mathrm{g} / \mathrm{l})$ & $1.885(1.143-3.109)$ & $0.013^{\mathrm{a}}$ & $2.824(1.605-4.969)$ & $<0.001^{\mathrm{a}}$ \\
\hline$\gamma \mathrm{GGT}(\geq 60 \mathrm{U} / 1 \mathrm{vs} .<60 \mathrm{U} / \mathrm{l})$ & $1.304(0.789-2.155)$ & 0.301 & $1.760(0.989-3.133)$ & 0.054 \\
\hline Child-Pugh score (A vs. B) & $1.200(0.546-2.638)$ & 0.649 & $1.294(0.551-3.038)$ & 0.554 \\
\hline Liver cirrhosis (no vs. yes) & $0.571(0.271-1.203)$ & 0.140 & $0.539(0.229-1.266)$ & 0.156 \\
\hline Tumor size ( $\geq 5 \mathrm{~cm}$ vs. $<5 \mathrm{~cm})$ & $1.190(0.716-1.975)$ & 0.502 & $1.722(0.958-3.096)$ & 0.069 \\
\hline Tumor number (multiple vs. single) & $1.344(0.741-2.438)$ & 0.330 & $1.736(0.922-3.266)$ & 0.087 \\
\hline Vascular invasion (yes vs. no) & $1.181(0.688-2.027)$ & 0.546 & $1.060(0.579-1.941)$ & 0.850 \\
\hline Lymphatic metastasis (yes vs. no) & $1.848(1.119-3.053)$ & $0.016^{\mathrm{a}}$ & $2.838(1.614-4.990)$ & $<0.001^{\mathrm{a}}$ \\
\hline Tumor encapsulation (complete vs. none) & $0.805(0.383-1.694)$ & 0.568 & $0.589(0.233-1.486)$ & 0.262 \\
\hline Tumor differentiation (poor vs. moderate/well) & $0.677(0.395-1.161)$ & 0.155 & $0.604(0.326-1.121)$ & 0.110 \\
\hline UBE2M (high vs. low) & $1.185(1.084-3.038)$ & $0.023^{\mathrm{a}}$ & $2.470(1.372-4.445)$ & $0.003^{\mathrm{a}}$ \\
\hline
\end{tabular}

$\mathrm{B}$, Multivariate analysis

\begin{tabular}{|c|c|c|c|c|}
\hline \multirow[b]{2}{*}{ Variable } & \multicolumn{2}{|l|}{ RFS } & \multicolumn{2}{|l|}{$\mathrm{OS}$} \\
\hline & HR $(95 \% \mathrm{CI})$ & P-value & HR $(95 \% \mathrm{CI})$ & P-value \\
\hline HBsAg (positive vs. negative) & $0.365(0.198-0.672)$ & $0.001^{\mathrm{a}}$ & $0.280(0.135-0.578)$ & $0.001^{\mathrm{a}}$ \\
\hline Serum CEA $(\geq 5 \mu \mathrm{g} / \mathrm{l}$ vs. $<5 \mu \mathrm{g} / \mathrm{l})$ & $1.348(0.788-2.034)$ & 0.275 & $2.051(1.108-3.797)$ & $0.022^{\mathrm{a}}$ \\
\hline Lymphatic metastasis (yes vs. no) & $1.518(0.895-2.577)$ & 0.122 & $1.891(1.038-3.442)$ & $0.037^{\mathrm{a}}$ \\
\hline UBE2M (high vs. low) & $1.892(1.11-3.214)$ & $0.018^{\mathrm{a}}$ & $2.842(1.151-5.332)$ & $0.001^{\mathrm{a}}$ \\
\hline
\end{tabular}

aP $<0.05$. Cox proportional hazards regression model. AFP, $\alpha$-fetoprotein; ALT, alanine aminotransferase; CA 19-9, carbohydrate antigen 19-9; CEA, carcinoembryonic antigen; CI, confidential interval; $\gamma$ GGT, $\gamma$-glutamyltranspeptidase; HBsAg, hepatitis B surface antigen; HR, hazard ratio; OS, overall survival; RFS, recurrence-free survival; UBE2M, neural precursor cell expressed developmentally downregulated 8-conjugating enzyme E2M.

cat. no. ab85830; Abcam); chromatin-licensing and DNA replication factor 1 (CDT1; 1:1,000; cat. no. ab202067; Abcam); poly (ADP-ribose) polymerase (PARP; 1:1,000; cat. no. ab74290; Abcam); cleaved (c)PARP (1:1,000; cat. no. ab4830; Abcam; caspase-3 (1:1,000; cat. no. ab197202; Abcam); caspase-9 (1:1,000; cat. no. ab219590; Abcam); cullin 1 (1:1,000; cat. no. ab75817; Abcam); and $\gamma$-H2A histone family member X ( $\gamma$-H2AX; 1:1,000; cat. no. ab26350; Abcam). Secondary horseradish peroxidase-conjugated antibodies against rabbit immunoglobulin G (IgG; 1:5,000; cat. no. sc-2004) or mouse IgG (1:5,000; cat. no. sc-2005; both Santa Cruz Biotechnology, Inc.) were then used at room temperature for $2 \mathrm{~h}$. Bands were visualized using Pierce ${ }^{\mathrm{TM}}$ ECL Western Blotting Substrate (Thermo Fisher Scientific, Inc.).
In vivo mouse xenograft study. The present study followed animal handling and experimental procedures, and was approved by the Animal Care and Use Committee of Lanzhou University Second Hospital (permit no. 2019A-019). Following anesthesia via $1.9 \%$ ether inhalation for $5 \mathrm{~min}$ (anesthesia was monitored based on the respiratory rate), 5-week-old male athymic nude mice $(n=14,20-35 \mathrm{~g}$; housed in an animal room maintained at $20-22^{\circ} \mathrm{C}$ and $30-70 \%$ relative humidity under a 12:12-h light/dark cycle) were subcutaneously injected with QBC939 cells in the groin

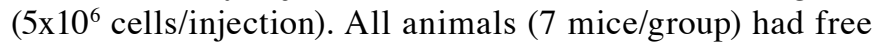
access to standard food and drinking water. After 3 days, the tumor-bearing mice were randomly divided into shNC and shUBE2M groups. Lentivirus was intratumorally injected 
A
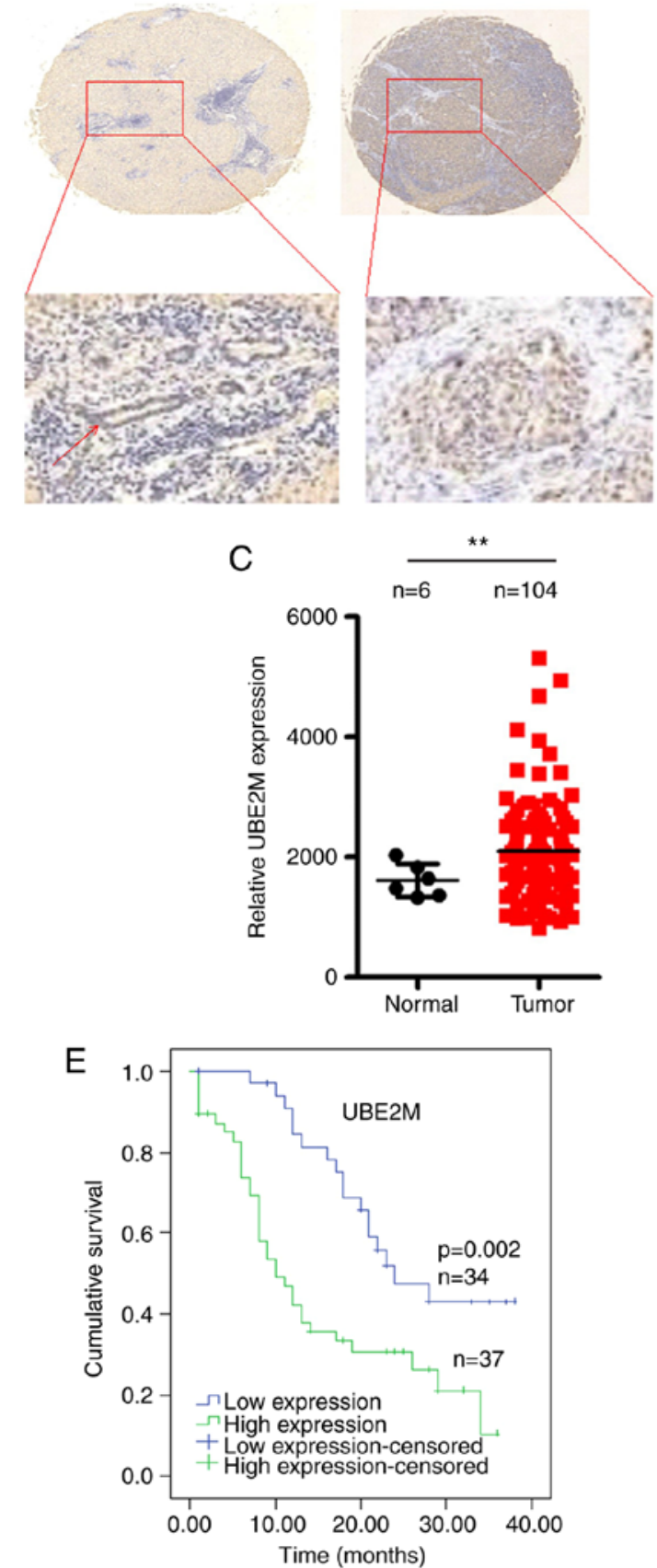

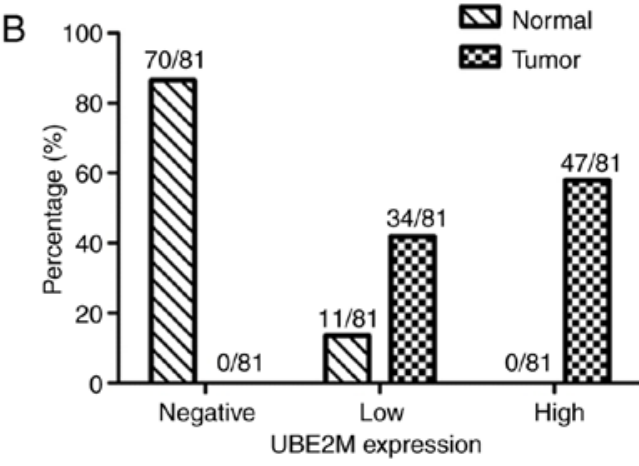

$\mathrm{D}$
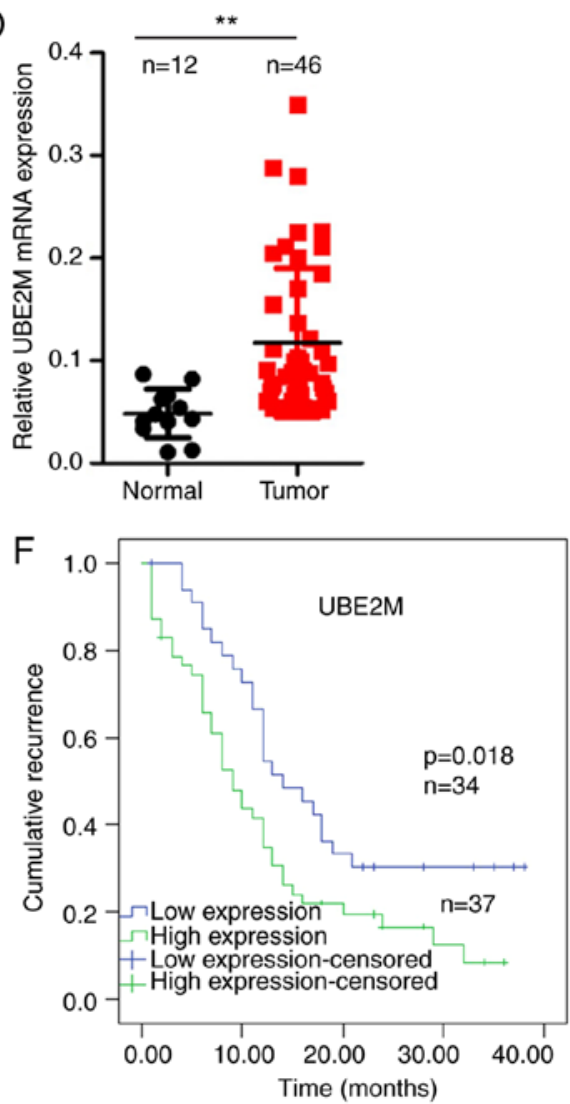

Figure 1. Expression and prognostic value of UBE2M in ICC. (A) Immunohistochemistry staining of human ICC tissue specimens using specific antibodies for UBE2M. (B) Quantification of UBE2M expression intensity. (C) UBE2M was overexpressed in ICC tumor tissues in the GSE26566 dataset. Among 104 clinical samples, increased expression of UBE2M was detected in tumor tissue compared with 6 normal intrahepatic bile duct tissue samples. (D) Relative expression of UBE2M was determined by reverse transcription-quantitative PCR in 46 ICC tumor tissues and 12 normal intrahepatic bile duct tissues. Kaplan-Meier curves for (E) overall survival and $(\mathrm{F})$ recurrence-free survival of patients with ICC, according to the expression of UBE2M. ${ }^{* *} \mathrm{P}<0.01$. ICC, intrahepatic cholangiocarcinoma; UBE2M, neural precursor cell expressed developmentally downregulated 8-conjugating enzyme E2M.

in mice $\left(5 \times 10^{8}\right.$ copies per injection). Vernier calipers were used to measure the tumor size once every other day. At 7 weeks later, the mice were sacrificed via cervical dislocation (confirmed by the cessation of breathing and heartbeat, and loss of nerve reflexes), and tumor xenografts were collected, weighed and photographed. Tumor tissues fixed with $10 \%$ formalin neutral buffer solution for $24 \mathrm{~h}$ in room temperature were paraffin-embedded and sectioned (4-5 $\mu \mathrm{m}$ thickness) for IHC. Paraffin sections were deparaffinized in $100 \%$ xylene and rehydrated in a graded ethanol series, and then peroxidase blocking with $1 \% \mathrm{H}_{2} \mathrm{O}_{2}$ for
$30 \mathrm{~min}$ at room temperature and antigen retrieval were performed. Sections were incubated overnight at $4^{\circ} \mathrm{C}$ with primary antibody against Ki-67 (1:100; cat. no. ab15580; Abcam), followed by incubation for $15 \mathrm{~min}$ at room temperautere with a biotin-labeled secondary antibody (1:5,000; Product \# 65-6140; Invitrogen) and subsequently with streptavidin-conjugated horseradish peroxidase (Fuzhou Maixin Biotech Co., Ltd.). DAB was applied to develop the peroxidase reaction. Then the sections were counterstained with hematoxylin (Fisher CS401-D) for $20 \mathrm{sec}$ at room temperature, mounted in neutral gum and analyzed 
using a Optiphot microscope (magnification, x200; Nikon Corporation) in five random fields per sample.

GSE dataset analyses. Public data for ICC [GSE26566 (20), containing 104 cases of ICC, 59 cases of matched non-cancerous livers and 6 cases of normal intrahepatic bile duct] were obtained from Gene Expression Omnibus (GEO) database (21).

Protein-protein interaction (PPI) network analyses. The STRING database (version 11; https://string-db. org/cgi/input.pl) was used to construct the PPI network of UBE2M (22).

Statistical analyses. Data were presented as the mean \pm standard deviation of three or more independent repeats. Comparisons between two groups were performed using Student's t-test, Fisher's exact test or $\chi^{2}$ test. Kaplan-Meier analysis and log-rank test, or univariate and multivariate analyses were used to analyze associations between TTR and overall survival and prognostic factors as appropriate. All statistical analysis were performed using SPSS software 18.0 (SPSS, Inc.). $\mathrm{P}<0.05$ was considered to indicate a statistically significant difference.

\section{Results}

UBE2M protein is overexpressed in ICC tissue specimens and associated with clinical outcomes. To evaluate the expression of UBE2M in ICC tissues, UBE2M expression was measured via IHC staining in tissue specimens derived from 81 pairs of primary ICC tissues and adjacent normal tissues. It was revealed that UBE2M was overexpressed in ICC tumor tissue compared with matched non-tumor tissue (Fig. 1A and B).

To further investigate the effects of UBE2M on ICC progression, UBE2M protein expression in ICC tumors from the GSE26566 dataset was analyzed; as presented in Fig. 1C, UBE2M protein was significantly upregulated in ICC tumor tissues compared with normal intrahepatic bile duct in the dataset $(\mathrm{P}<0.01)$. RT-qPCR analysis revealed that UBE2M mRNA was significantly overexpressed in ICC tumor tissue compared with normal intrahepatic bile duct tissues (Fig. 1D). These findings indicated that UBE2M was overexpressed in ICC tumor tissues compared with in normal tissues.

To demonstrate the prognostic relevance of UBE2M expression in ICC, patients were separated into high (strong or moderate intensities) or low (weak or negative intensities) expression groups according to IHC staining of ICC tumor tissues. The expression of UBE2M was not significantly associated with any of the assessed clinicopathological variables (Table I). Kaplan-Meier analysis revealed that the overall survival of patients with high expression of UBE2M was significantly reduced compared with those with low expression ( $\mathrm{P}=0.002$; Fig. 1E). The TTR of patients with high UBE2M expression (median, 9 months) was significantly shorter than those with low expression (median, 14 months; $\mathrm{P}=0.018$; Fig. 1F). Univariate analysis revealed that high expression of UBE2M was clearly associated with worse prognosis and reduced TTR in patients with ICC (Table II). Furthermore, multivariate Cox proportional hazards regression analysis was performed for several clinicopathological factors (Table II). It was demonstrated that that high expression of UBE2M $(\mathrm{P}<0.001)$, negative hepatitis $\mathrm{B}$ surface antigen (HBsAg; $\mathrm{P}<0.001$ ), high expression of serum carcinoembryonic antigen $(\mathrm{CEA} ; \mathrm{P}<0.05)$ and lymphatic metastasis $(\mathrm{P}<0.05)$ were independent prognostic factors for poor overall survival. Additionally, multivariate regression analysis showed that UBE2M overexpression and positive $\mathrm{HBsAg}$ were independent factors for recurrence-free survival $(\mathrm{P}=0.018$ and $\mathrm{P}<0.001$, respectively).

UBE2M knockdown inhibits the growth of QBC939 cells and HUCCT cells. To further determine the role of UBE2M in ICC tumor cell lines, shUBE2M was used to knock down UBE2M expression in the ICC cell lines QBC939 and HUCCT1. As shown in Fig. 2C, western blotting revealed that UBE2M shRNA markedly downregulated expression of UBE2M protein in QBC939 and HUCCT1 cells (Fig. 2C). Subsequently, the effects of UBE2M knockdown on the viability and colony formation of these ICC cells were evaluated; CCK-8 analysis revealed that the viability of shUBE2M cells was significantly inhibited compared with that of shNC cells $(\mathrm{P}<0.001$; Fig. 2A). Similarly, colony formation was significantly decreased in shUBE2M cells $(\mathrm{P}<0.001$; Fig. $2 \mathrm{~B})$. These data indicated that the UBE2M suppression resulted in the inhibition of ICC cell viability.

As UBE2M is an important component of neddylation, the expression of the cullin substrates ORC1 and CDT1 were subsequently investigated. Immunoblotting revealed that the expression levels of cullin 1 in ICC cell lines were not notably changed, whereas NEDD8-cullin1 expression was notably reduced following UBE2M knockdown. The cullin substrates ORC1 and CDT1, whose overexpression induce DNA damage responses, were clearly upregulated in shUBE2M cells. Additionally, upregulation of $\gamma-\mathrm{H} 2 \mathrm{AX}$, a marker of DNA double strand breaks (DSBs), was observed in the shUBE2M groups (Fig. 2C).

UBE2M knockdown induces ICC tumor cell apoptosis. The previous findings indicated that UBE2M silencing suppressed the viability and proliferation of ICC tumor cells. To further investigate the potential mechanisms involved, the expression of apoptosis-associated proteins was evaluated, including PARP, cPARP, caspase-3, and caspase-9. UBE2M knockdown appeared to induce apoptosis as demonstrated by a shrunk cellular morphology (Fig. 3A), the upregulation of cPARP, and caspase-3 and -9 (Fig. 3C), and the appearance of a sub-G1 peak (Fig. 3B).

Effects of UBE2M knockdown on mouse xenograft growth. Due to the antitumor effects of UBE2M knockdown on ICC cells in vitro, its effects were further examined in nude mouse xenografts. Tumor-bearing nude mice were intratumorally injected with lenti-shUBE2M, and then tumor growth was monitored. UBE2M knockdown in reduced growth of tumors, with significantly decreased tumor volume $(\mathrm{P}<0.01$; Fig. $4 \mathrm{~A})$ and weight $(\mathrm{P}<0.01$; Fig. 4B) compared with the control group injected with lenti-shNC. IHC staining demonstrated that injection of lenti-shUBE2M led to a marked reduction of Ki67 expression, a marker of proliferation, in nude mouse xenografts (Fig. 4C). 
A

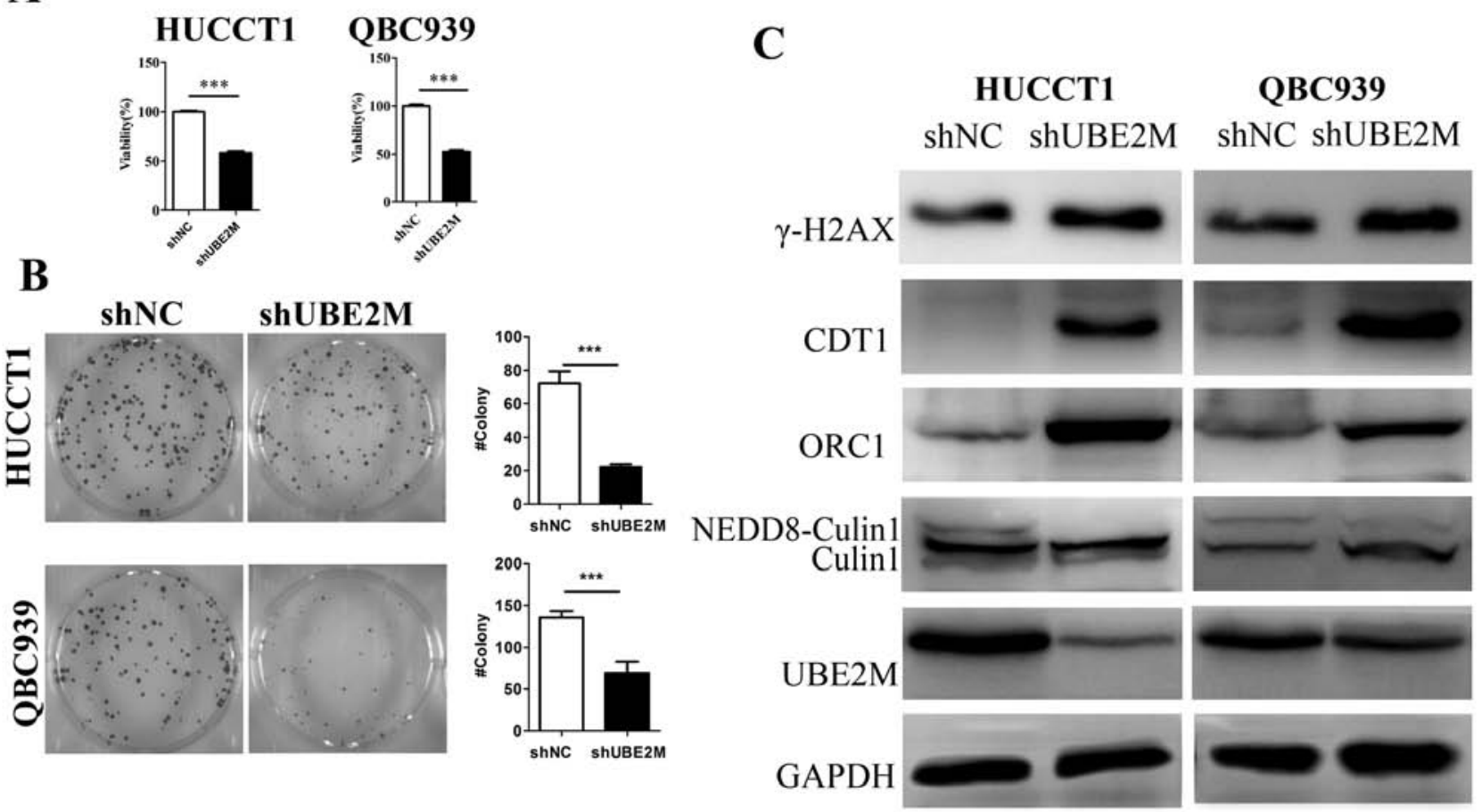

Figure 2. Effects of UBE2M knockdown on the viability and colony formation of intrahepatic cholangiocarcinoma cells. (A) Effects of UBE2M knockdown on the viability of HUCCT1 and QBC939 cells. Cells were transfected with shUBE2M for $48 \mathrm{~h}$, and the viability was assessed by Cell Counting Kit-8 assays (n=3). (B) Effects of UBE2M knockdown on clonogenic survival. HUCCT1 and QBC939 cells were transfected with shUBE2M for 48 h (n=3). (C) Effects of UBE2M knockdown on the neddylation of cullin 1, and the expression of its substrates in HUCCT1 and QBC939 cells. Knockdown of UBE2M induced accumulation of the cullin-RING ligase substrates ORC1, CDT1, $\gamma$-H2AX. Data are presented as the mean $\pm \mathrm{SD}$. ${ }^{* * *} \mathrm{P}<0.001$. CDT1, chromatin-licensing and DNA replication factor 1; $\gamma$-H2AX, $\gamma$-H2A histone family member X; NC, negative control; NEDD8, neural precursor cell expressed developmentally downregulated 8; ORC1, origin recognition complex subunit 1; sh, short hairpin (RNA); UBE2M, NEDD8-conjugating enzyme E2M.

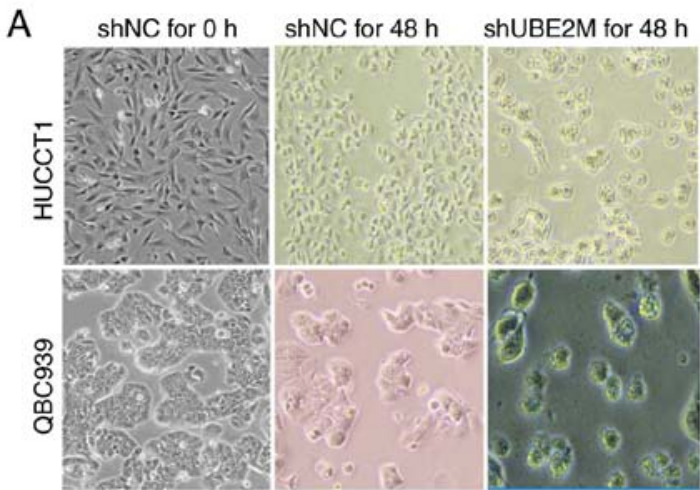

B

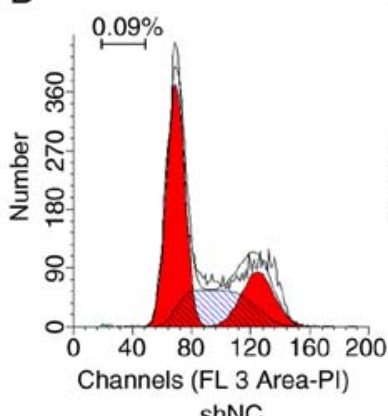

HUCCT1

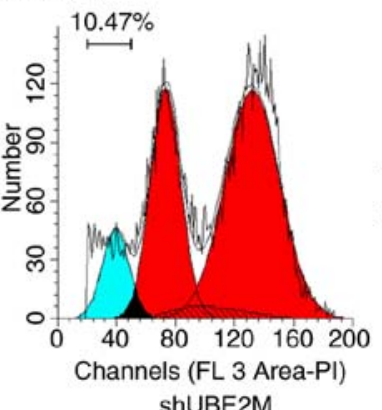

ShUBE2M

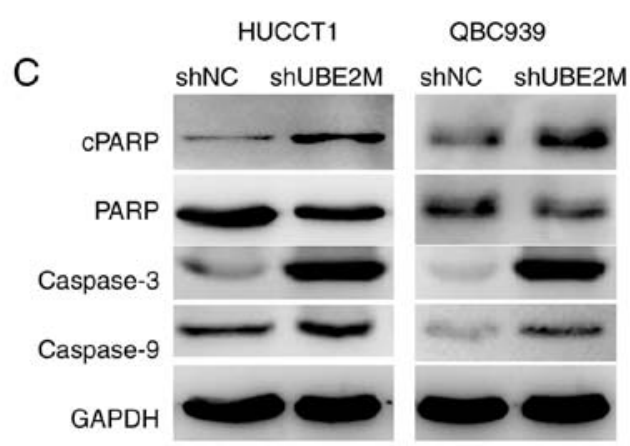

QBC939

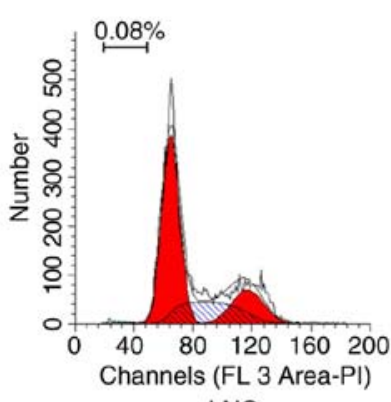

shNC

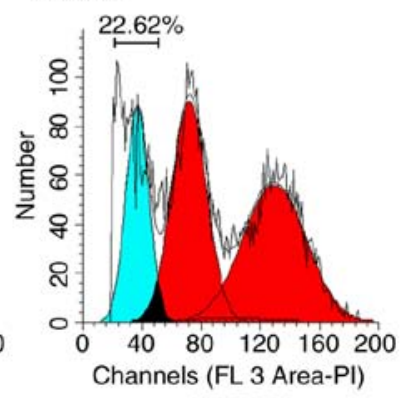

ShUBE2M

Figure 3. UBE2M knockdown induces intrahepatic cholangiocarcinoma tumor cell apoptosis. Knockdown of UBE2M induced apoptosis and the expression of proapoptotic proteins in HUCCT1 and QBC939 cells. Cells were transfected with shUBE2M for $48 \mathrm{~h}$ and then subjected to (A) morphological observation or (B) PI staining and FACS analysis. (C) Western blot analysis of apoptosis-associated proteins; GAPDH was used as a loading control. NC, negative control; cPARP, cleaved poly (ADP-ribose) polymerase; sh, short hairpin (RNA); UBE2M, neural precursor cell expressed developmentally downregulated 8-conjugating enzyme E2M. 
A

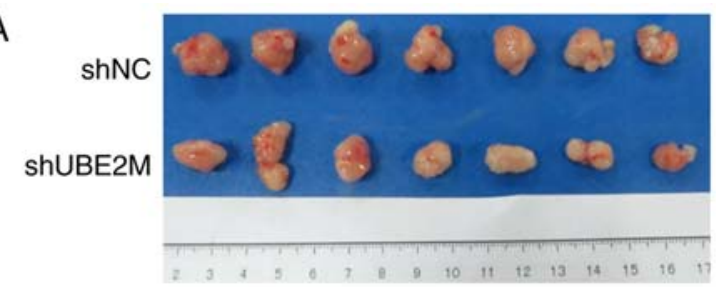

B

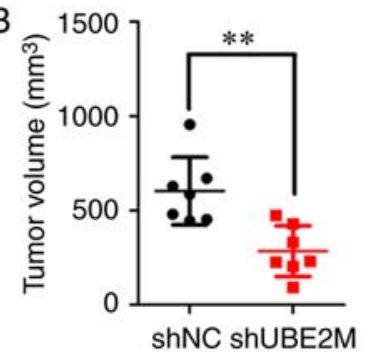

D

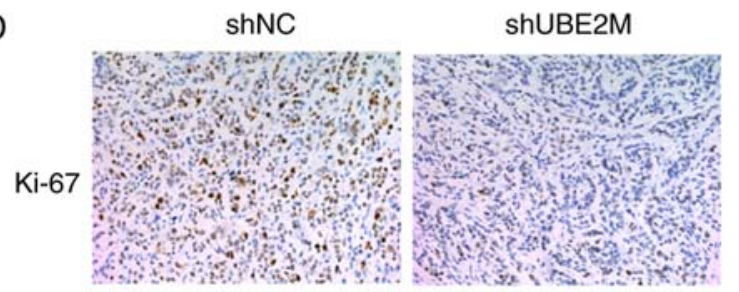

Figure 4. UBE2M knockdown inhibits the growth of intrahepatic cholangiocarcinoma cell xenografts. (A) Images of shNC and shUBE2M xenograft tumors were assessed 7 weeks after initiation of the experiment $(n=7)$. Tumor tissues of mice were collected, photographed, weighed and stored for further analyses. (B) Tumor volume and (C) weight were reduced following UBE2M knockdown. (D) Immunostaining revealed a decrease in Ki-67 expression. ${ }^{* *} \mathrm{P}<0.01$. NC, negative control; sh, short hairpin (RNA); UBE2M, neural precursor cell expressed developmentally downregulated 8-conjugating enzyme E2M.

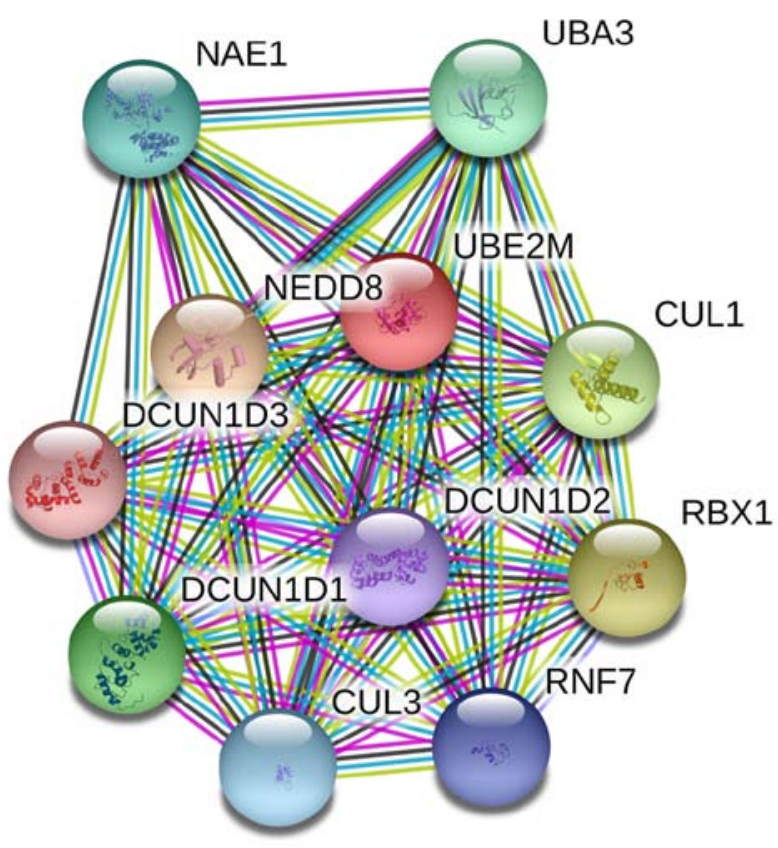

Figure 5. Protein-protein interaction network for UBE2M predicted by the STRING database. CUL, cullin; DCUN, defective in cullin neddylation; NAE1, NEDD8 activating enzyme E1; NEDD8, neural precursor cell expressed developmentally downregulated 8; RBX1, RING-box protein 1; RNF7, ring finger protein 7; UBA3, ubiquitin-like modifier activating enzyme 3; UBE2M, NEDD8-conjugating enzyme E2M.
Finally, protein-protein interaction (PPI) network analysis was performed using the STRING database, based on previous studies into UBE2M function (13,23-25). As presented in Fig. 5, UBE2M was revealed to interact with CUL1, CUL3, defective in cullin neddylation 1 (DCUN1) D1, DCUN1D2, DCUN1D3, NAE1, NEDD8, RING-box protein 1 , ring finger protein 7 and ubiquitin-like modifier activating enzyme 3 .

\section{Discussion}

ICC represents the second most common hepatic carcinoma, with a majority of patients diagnosed at a nonsurgical stage; even following early radical resection, the rate of recurrence after surgery is $60-70 \%$ (26). Therefore, postoperative adjuvant therapy is often considered, and the development of effective treatment strategies is urgently required. As one of the critical molecular pathways involved in carcinogenesis and the progression of various cancers, including lung, breast, bladder, gastric and prostate cancers (27-31), the neddylation pathway was also upregulated in ICC (14). Neddylation modification and CRL E3 ligase are attractive anticancer targets (32-34). An inhibitor of NEDD8-activating enzyme E1, MLN4924, was demonstrated to suppress the growth of ICC tumor cells both in vitro and in vivo (14); however, there has been limited investigation into the effects of Nedd8-conjugating enzyme UBE2M.

In the present study, it was reported for the first time, to the best of our knowledge, that UBE2M protein was overexpressed in ICC tissue specimens compared with corresponding normal tissues, consistent to with previous studies in other types of human cancers, including lung cancer, and head and neck squamous cell carcinoma $(27,35)$. However, the imbalanced group numbers in the GSE26566 dataset, including 104 cases of ICC and 6 cases of normal intrahepatic bile duct, may impact the interpretation of these results. More normal intrahepatic bile duct samples should be collected for further analysis in future studies. Overexpression of UBE2M was previously associated with worse prognosis in lung cancer (27), suggesting that UBE2M expression may be a novel prognostic marker for patients with ICC. In the study, it was demonstrated that the expression of UBE2M was not significantly associated with any assessed clinicopathologic features in patients with ICC, which may be due to the small sample size; a previous study reported that Neuropilin-1 expression was not significantly associated with clinicopathologic characteristics in breast cancer tissues as a result of the small number of patients enrolled in this study (36).

Silencing of UBE2M expression inhibited ICC cell growth and led to apoptosis. Molecularly, knockdown of UBE2M expression blocked the second step of neddylation pathway in HUCCT1 cells and QBC939 cells, leading to accumulation of cullin substrates ORC1 and CDT1, and subsequently resulting in DSBs and the induction DNA damage responses, as revealed by the upregulated expression of $\gamma$-H2AX. UBE2M silencing results in increased DNA breakages and sensitivity to DNA-damaging agents (24). Additionally, aberrant DNA repair and increased apoptosis was observed, as determined by low expression of PARP, which is involved in DNA repair (37) and apoptosis (38), and increased cPARP, caspase-3 
and caspase-9 following UBE2M knockdown (11). Induction of cellular apoptosis is regarded as an important antitumor mechanism; various stresses, including antitumor gene activation (39), telomere dysfunction $(40,41)$ and DNA damage (42) can all initiate cellular apoptosis.

Mechanistically, UBE2M knockdown resulted in the reduced viability and colony formation of ICC cell lines, potentially due to DNA damage responses and apoptosis. UBE2M knockdown not only inhibited the colony formation of ICC cells in vitro, but also suppressed ICC tumor growth in vivo, as determined by the reduced size of tumor xenografts infected with the shUBE2M lentivirus.

Additionally, protein-protein interaction (PPI) network analysis was performed for UBE2M to identify molecules potentially involved in its effects. In future experiments, gene expression or RNA sequencing followed by gene set enrichment analysis on cell lines will be performed pre- and post-shUBE2M treatment to identify the relevant molecular pathways involved in the development of ICC.

In conclusion, the present study revealed that UBE2M served an important role in ICC progression, and that targeting UBE2M protein is a potential anticancer strategy for ICC. Further investigation of the underlying mechanisms of UBE2M in ICC is required.

\section{Acknowledgements}

Not applicable.

\section{Funding}

This work was supported by Medical Research Project of Lanzhou Chengguan District, China (grant no. 2018-01-11).

\section{Availability of data and materials}

The datasets used and analyzed during the current study are available from the corresponding author on reasonable request.

\section{Authors' contributions}

BZ, CG and ZJ conceived the study, carried out the overall experimental design and data interpretation, and prepared and revised the manuscript. BZ performed the majority of the experiments. CG, DS and JM performed immunohistochemical assays. JZ and LG performed the colony formation assay. JG performed the western blot analysis. All authors have read and approved the final version of this manuscript.

\section{Ethics approval and consent to participate}

The human study was approved by the Lanzhou University Second Hospital Ethics Committee, and informed consent was obtained from each patient. The animal study was approved by the Animal Care and Use Committee of Lanzhou University Second Hospital.

\section{Patient consent for publication}

Not applicable.

\section{Competing interests}

The authors declare that they have no competing interests.

\section{References}

1. Bedford L, Lowe J, Dick LR, Mayer RJ and Brownell JE: Ubiquitin-like protein conjugation and the ubiquitin-proteasome system as drug targets. Nat Rev Drug Discov 10: 29-46, 2011.

2. O'Connor HF and Huibregtse JM: Enzyme-substrate relationships in the ubiquitin system: Approaches for identifying substrates of ubiquitin ligases. Cell Mol Life Sci 74: 3363-3375, 2017.

3. Petroski MD and Deshaies RJ: Function and regulation of cullin-RING ubiquitin ligases. Nat Rev Mol Cell Biol 6: 9-20, 2005.

4. Deshaies RJ, Emberley ED and Saha A: Control of cullin-ring ubiquitin ligase activity by nedd8. Subcell Biochem 54: 41-56, 2010.

5. Ying J, Zhang M, Qiu X and Lu Y: Targeting the neddylation pathway in cells as a potential therapeutic approach for diseases. Cancer Chemother Pharmacol 81: 797-808, 2018.

6. Xirodimas DP: Novel substrates and functions for the ubiquitin-like molecule NEDD8. Biochem Soc Trans 36: 802-806, 2008.

7. Zuo W, Huang F, Chiang YJ, Li M, Du J, Ding Y, Zhang T, Lee HW, Jeong LS, Chen Y, et al: C-Cbl-mediated neddylation antagonizes ubiquitination and degradation of the TGF- $\beta$ type II receptor. Mol Cell 49: 499-510, 2013.

8. Zhao Y, Morgan MA and Sun Y: Targeting neddylation pathways to inactivate cullin-RING ligases for anticancer therapy. Antioxid Redox Signal 21: 2383-2400, 2014.

9. Watson IR, Irwin MS and Ohh M: NEDD8 pathways in cancer, sine quibus non. Cancer Cell 19: 168-176, 2011.

10. Huang DT, Ayrault O, Hunt HW, Taherbhoy AM, Duda DM, Scott DC, Borg LA, Neale G, Murray PJ, Roussel MF and Schulman BA: E2-RING expansion of the NEDD8 cascade confers specificity to cullin modification. Mol Cell 33: 483-495, 2009.

11. Cukras S, Morffy N, Ohn T and Kee Y: Inactivating UBE2M impacts the DNA damage response and genome integrity involving multiple cullin ligases. PLoS One 9: e101844, 2014.

12. Xu B, Deng Y, Bi R, Guo H, Shu C, Shah NK, Chang J, Liu G, Du Y, Wei W and Wang C: A first-in-class inhibitor, MLN4924 (pevonedistat), induces cell-cycle arrest, senescence, and apoptosis in human renal cell carcinoma by suppressing UBE2M-dependent neddylation modification. Cancer Chemother Pharmacol 81: 1083-1093, 2018.

13. Zhou W, Xu J, Tan M, Li H, Li H, Wei W and Sun Y: UBE2M is a stress-inducible dual E2 for neddylation and ubiquitylation that promotes targeted degradation of UBE2F. Mol Cell 70: 1008-1024, 2018.

14. Gao Q, Yu GY, Shi JY, Li LH, Zhang WJ, Wang ZC, Yang LX, Duan M, Zhao H, Wang XY, et al: Neddylation pathway is up-regulated in human intrahepatic cholangiocarcinoma and serves as a potential therapeutic target. Oncotarget 5: 7820-7832, 2014.

15. Altekruse SF, Devesa SS, Dickie LA, McGlynn KA and Kleiner DE: Histological classification of liver and intrahepatic bile duct cancers in SEER registries. J Registry Manag 38: 201-205, 2011.

16. Bertuccio P, Bosetti C, Levi F, Decarli A, Negri E and La Vecchia C: A comparison of trends in mortality from primary liver cancer and intrahepatic cholangiocarcinoma in Europe. Ann Oncol 24: 1667-1674, 2013.

17. Bridgewater J, Galle PR, Khan SA, Llovet JM, Park JW, Patel T, Pawlik TM and Gores GJ: Guidelines for the diagnosis and management of intrahepatic cholangiocarcinoma. J Hepatol 60: 1268-1289, 2014.

18. Poultsides GA, Zhu AX, Choti MA and Pawlik TM: Intrahepatic cholangiocarcinoma. Surg Clin North Am 90: 817-837, 2010.

19. Livak KJ and Schmittgen TD: Analysis of relative gene expression data using real-time quantitative PCR and the 2(-Delta Delta C(T)) method. Methods 25: 402-408, 2001.

20. Andersen JB, Spee B, Blechacz BR, Avital I, Komuta M, Barbour A, Conner EA, Gillen MC, Roskams T, Roberts LR, et al: Genomic and genetic characterization of cholangiocarcinoma identifies therapeutic targets for tyrosine kinase inhibitors. Gastroenterology 14: 1021-1031, 2012. 
21. Barrett T, Wilhite SE, Ledoux P, Evangelista C, Kim IF Tomashevsky M, Marshall KA, Phillippy KH, Sherman PM, Holko M, et al: NCBI GEO: Archive for functional genomics data sets-update. Nucleic Acids Res 41: D991-D995, 2013.

22. Szklarczyk D, Gable AL, Lyon D, Junge A, Wyder S, Huerta-Cepas J, Simonovic M, Doncheva NT, Morris JH, Bork P, et al: STRING v11: Protein-protein association networks with increased coverage, supporting functional discovery in genome-wide experimental datasets. Nucleic Acids Res 47: D607-D613, 2019.

23. Li L, Kang J, Zhang W, Cai L, Wang S, Liang Y, Jiang Y, Liu X, Zhang Y, Ruan $\mathrm{H}$, et al: Validation of NEDD8-conjugating enzyme UBC12 as a new therapeutic target in lung cancer. EBioMedicine 45: 81-91, 2019.

24. Brown JS, Lukashchuk N, Sczaniecka-Clift M, Britton S, le Sage C, Calsou P, Calsou P, Beli P, Galanty Y and Jackson SP: Neddylation promotes ubiquitylation and release of $\mathrm{ku}$ from DNA-damage sites. Cell Rep 11: 704-714, 2015.

25. Jimeno S, Fernandez-Avila MJ, Cruz-Garcia A, Cepeda-Garcia C, Gomez-Cabello D and Huertas P: Neddylation inhibits CtIP-mediated resection and regulates DNA double strand break repair pathway choice. Nucleic Acids Res 43: 987-999, 2015.

26. Mavros MN, Economopoulos KP, Alexiou VG and Pawlik TM: Treatment and prognosis for patients with intrahepatic cholangiocarcinoma: Systematic review and meta-analysis. JAMA Surg 149: 565-574, 2014.

27. Li L, Wang M, Yu G, Chen P, Li H, Wei D, Zhu J, Xie L, Jia H, Shi J, et al: Overactivated neddylation pathway as a therapeutic target in lung cancer. J Natl Cancer Inst 106: dju083. 2014.

28. Wang W, Liu Z, Qu P, Zhou Z, Zeng Y, Fan J, Liu Y, Guo Y and Qiu J: Knockdown of regulator of cullins-1 (ROC1) expression induces bladder cancer cell cycle arrest at the G2 phase and senescence. PLoS One 8: e62734, 2013.

29. Lan H, Tang Z, Jin H and Sun Y: Neddylation inhibitor MLN4924 suppresses growth and migration of human gastric cancer cells. Sci Rep 6: 24218, 2016.

30. Wen M, Kwon Y, Wang Y, Mao JH and Wei G: Elevated expression of UBE2T exhibits oncogenic properties in human prostate cancer. Oncotarget 6: 25226-25239, 2015.

31. Ueki T, Park JH, Nishidate T, Kijima K, Hirata K, Nakamura Y and Katagiri T: Ubiquitination and downregulation of BRCA1 by ubiquitin-conjugating enzyme E2T overexpression in human breast cancer cells. Cancer Res 69: 8752-8760, 2009.
32. $\mathrm{Wu} \mathrm{S}$ and $\mathrm{Yu} \mathrm{L}$ : Targeting cullin-RING ligases for cancer treatment: Rationales, advances and therapeutic implications. Cytotechnology 68: 1-8, 2016.

33. Jia L, Soengas MS and Sun Y: ROC1/RBX1 E3 ubiquitin ligase silencing suppresses tumor cell growth via sequential induction of G2-M arrest, apoptosis, and senescence. Cancer Res 69: 4974-4982, 2009.

34. Zhou L, Zhang W, Sun Y and Jia L: Protein neddylation and its alterations in human cancers for targeted therapy. Cell Signal 44 : 92-102, 2018

35. Zhang W, Liang Y, Li L, Wang X, Yan Z, Dong C, Zeng MS, Zhong Q, Liu XK, Yu J, et al: The nedd8-activating enzyme inhibitor MLN4924 (TAK-924/Pevonedistat) induces apoptosis via c-myc-noxa axis in head and neck squamous cell carcinoma. Cell Prolif 52: e12536, 2019.

36. Seifi-Alan M, Shams R, Bandehpour M, Mirfakhraie R and Ghafouri-Fard S: Neuropilin-1 expression is associated with lymph node metastasis in breast cancer tissues. Cancer Manag Res 10: 1969-1974, 2018.

37. Hosoya $\mathrm{N}$ and Miyagawa K: Targeting DNA damage response in cancer therapy. Cancer Sci 105: 370-388, 2018.

38. Affar EB, Germain M, Winstall E, Vodenicharov M, Shah RG, Salvesen GS and Poirier GG: Caspase-3-mediated processing of poly(ADP-ribose) glycohydrolase during apoptosis. J Biol Chem 276: 2935-2942, 2001.

39. Pistritto G, Trisciuoglio D, Ceci C, Garufi A and D'Orazi G: Apoptosis as anticancer mechanism: Function and dysfunction of its modulators and targeted therapeutic strategies. Aging (Albany NY) 8: 603-619, 2016.

40. Chin L, Artandi SE, Shen Q, Tam A, Lee SL, Gottlieb GJ, Greider CW and DePinho RA: P53 deficiency rescues the adverse effects of telomere loss and cooperates with telomere dysfunction to accelerate carcinogenesis. Cell 97: 527-538, 1999.

41. Titen SW and Golic KG: Telomere loss provokes multiple pathways to apoptosis and produces genomic instability in Drosophila melanogaster. Genetics 180: 1821-1832, 2008.

42. Kee Y, Huang M, Chang S, Moreau LA, Park E, Smith PG and D'Andrea AD: Inhibition of the nedd8 system sensitizes cells to DNA interstrand cross-linking agents. Mol Cancer Res 10: 369-377, 2012. 